\title{
Isolation, Molecular Characterisation and Vaccine Effectiveness Study of Lumpy Skin Disease Virus in Selected Diary Farms of Central Ethiopia
}

\author{
Girma Zewdie $^{1 *} \quad$ Bedaso Mammo $^{2,3} \quad$ Esayas Gelaye $^{1} \quad$ Belayneh Getachew $^{1} \quad$ Berecha Bayssa $^{1}$ \\ 1. National Veterinary Institute, Debre-Zeit, Ethiopia, P. O. Box: 19
}

2.Addis Ababa University, college of Veterinary Medicine and Agriculture, Debre-Zeit, Ethiopia, P. O. Box: 34

3.University of Cambridge, Department of Microbiology, Immunology \& Veterinary Public Health

National Veterinary Institute, Debre-Zeit, Ethiopia. P.O. Box 19

\begin{abstract}
Lumpy skin disease (LSD) is economically important viral disease of cattle mainly in Africa and Middle East. Mass vaccination is a better strategy to control this disease in endemic countries. However, there is an increasing field report of vaccine failure. Thus, observational study was designed to investigate LSD outbreaks and vaccine effectiveness. Different dairy farms were selected on basis of reports of outbreak occurrence. A total of 191 dairy cattle from 17 smallholder and 1551 from four big dairy farms were included for assessment. Skin biopsies were taken from 31 LSD affected cattle for isolation and molecular characterization. Semi-structured questionnaires were administered to farm owners, animal health personnel and farm managers to gather relevant epidemiological data. Out of 1742 dairy cattle, $213(12.2 \%)$ showed characteristic clinical signs of LSD and 27 cattle (1.5\%) were died as the result of the disease. Viruses were isolated from 26 skin samples on Vero cell line and the isolates were confirmed to be LSDV using conventional PCR and real-time PCR. The complete sequence of RPO30 gene of the isolates showed that all isolates substituted at least one nucleotide in respect to vaccine strains. The vaccine effectiveness of annual vaccination was estimated to be $67.1 \%(95 \% \mathrm{CI}=57.3-74.7)$ in contrast to vaccination during outbreaks. Conversely, the occurrences of outbreak of LSD infection in vaccinated dairy herds are suggestive of vaccine failure. Thus, it's the time to direct efforts towards to the development of new effective vaccine.
\end{abstract}

Keywords: Dairy Cattle; Ethiopia; LSD; LSDV; Outbreak; Vaccine Effectiveness

DOI: $10.7176 / \mathrm{JBAH} / 9-11-01$

Publication date:June $30^{\text {th }} 2019$

\section{INTRODUCTION}

Lumpy skin disease (LSD) is a viral disease of cattle which is characterized by high fever, multiple circumscribed firm skin nodules, enlargement of superficial lymph nodes, nasal discharge and lachrymation; but in severity, clinical signs are highly variable. It is caused by Lumpy skin disease virus which is a member of the Capripoxvirus genus in the Poxviridae family. The virus is antigenically indistinguishable from other capripox viruses such as sheep pox virus (SPPV) and goat pox (GTPV) (Davies and Otema 1981; Woods 1988). LSDV mainly affects cattle and the disease is more severe in lactating and pregnant cows (Tuppurainen and Oura 2012).

Transmission has mostly been associated with blood-feeding insects with frequent feeding habits (Lefèvre and Gourreau, 2010; Chihota et al., 2001). Ticks have recently been implicated in the transmission of LSDV (Tuppurainen et al., 2011). The morbidity and mortality rates of LSD vary considerably, depending on host susceptibility and the abundance of insect vectors involved in transmission. The morbidity rates are generally between $1 \%$ and $20 \%$, but in few outbreak reports the morbidity rates exceeded $50 \%$. On the other hand, mortality of 10 to $40 \%$ and even higher have been reported on occasion but the lower range of 1 to $5 \%$ is more usual (Babiuk et al., 2008).

Lumpy skin disease was first observed in 1929 in northern Rhodesia (currently Zambia) and rapidly spread north and south of Africa (Tuppurainen and Oura 2012). The disease was then reported in out of Africa in different countries in the Middle East in late 1980s (Mohamed et al., 2014). It now appears to be spreading to new territories such as turkey and Iraq and threatens the entire European countries (EMPRES, 2013). LSD was first observed in Ethiopia in 1983 in the northwestern part of the country (Mebratu et al., 1984). It has now spread to almost all regions and agro-ecological zones and became endemic. Epidemic reoccurs after an interval of 5-6 years cycle in cattle population (Gari, 2011). The disease causes high economic losses through decreased milk production, emaciation and poor growth in infected animals, permanent damage to hides, abortion, temporary or permanent infertility, and sometimes death (Tuppurainen and Oura, 2012; Babiuk et al., 2008; Chihota et al., 2003). Moreover, it adversely affects the trade of livestock and livestock products. LSD is categorized by the World Organization for animal Health (OIE) as notifiable diseases due to their potential for rapid spread and substantial economic impact. On the other hand, the livelihood of more than $80 \%$ of population of Ethiopia is dependent on livestock. Therefore, LSD is one of the most economically important livestock diseases in the country. 
Vaccination is the effective method to control the disease in endemic countries like Ethiopia. The attenuated Kenyan sheep-and-goat pox vaccine strain KS-1 has been used to protect cattle against LSD in the country because of its advantage of conferring cross-protection to LSD (OIE, 2012). In contrary, field reports of LSD outbreak in vaccinated animals has been increasing during past years (Ayelet et al., 2014; Gelaye et al., 2015; Abutarbush, 2013; Brenner et al., 2009). Similar phenomenon has also reported from other countries (Abutarbush, 2013; Brenner et al., 2009). Furthermore, similar reports also came from different smallholder and big dairy farms following 2014/15 LSD outbreak eruption in Ethiopia and these demanded investigation of the situation.

As the result, the vaccine production companies and scholars are motivated to rule out the cause behind the current LSD vaccination failure. There is also a knowledge gap in the country to fully understand the existing incomplete protection of the vaccine. In addition, there are few studies on the type of the virus circulating in Ethiopia.

Therefore, the objectives of this study were:

- $\quad$ To isolate and characterize virulent field isolates of LSDV circulating in the area;

- To assess the current LSD vaccine effectiveness in the context of the existing vaccination strategy

- $\quad$ To investigate LSD outbreak in dairy cattle in the central parts of Ethiopia

\section{MATERIALS AND METHODS}

\subsection{Study Area}

Outbreak investigation was undertaken from September 2014 to January 2015 in the central parts of Ethiopia. Five towns from three Zones of Oromia Regional State; naming East Shoa (Bishoftu), North Shoa (Selale), West Shoa (Ambo, Holeta and Ginchi) and Akaki-Kaliti sub-city of Addis Ababa were purposively selected for the study based on LSD outbreak report. The altitude of the study areas ranges from $2391 \mathrm{~m}$ at Holeta town and 1,500-2,200 $\mathrm{m}$ at Bishoftu town above sea level. The areas exhibit a bimodal pattern of rainfall, with the highest rainfall concentration occurs from June to September and the short rainfall from March to April. The annual average rainfall of these areas is estimated from 1,150 and 2,200 $\mathrm{mm}$ and the annual mean daily temperature varies from $12.9{ }^{\circ} \mathrm{C}$ to $28.6{ }^{\circ} \mathrm{C}$ with moderate seasonal variation (NMSA, 2005).

\subsection{Study Animals and Farms}

All animals in the study were crossbreds with different exotic blood level and were kept for dairy and research purposes. Since the dairy farms were intended for milk production, study animals were largely cows and heifers. The farms included in the study were categorized as smallholder and big dairy farms based on herd size and management system. 17 smallholder dairy farms were included in this study. The smallholder dairy farms contain 2 to 30 dairy cattle and managed under intensive system and were usually known as urban dairy farms.

Four big dairy farms were also included in this study. These were Ethiopian Dairy and Meat Industry Development Institute (EDMIDI), Bishoftu; Ethiopian Institute of Agricultural Research (H-EIAR), Holeta; Ethiopian Institute of Agricultural Research (B-EIAR), Bishoftu and Selale Dairy Development Farms (SDDF). The big dairy farms hold more than 100 cattle each and provided with concentrate feeds and allowed to graze on farms owned pasture. However, SDDF uses concentrate feed and communal pasture for grazing. The big dairy farms separately managed young and adult animals. Big farms had their own animal health personnel to handle their animal health issues such as regular monitoring, treating and vaccinating animals. However, the smallholder dairy farms seek advice from veterinarians when their animals encounter serious health problem.

\subsection{Study Design}

The study areas were selected purposively based on reports of LSD outbreaks came to NVI. Three smallholder dairy farms from Akaki, seven from Bishoftu, two from Ambo and five from Ginchi were included to assess field LSD vaccine effectiveness. A total of 191 and 1551 dairy cattle were included in the study from 17 smallholder dairy farms and four big dairy farms respectively.

In addition, semi-structured questionnaires were administered to 25 purposively selected farm owners, veterinarians, animal health professionals and production managers to generate information. Information on vaccination history; clinical signs, age, sex, and breed of animals, management system, morbidity, and mortality was gathered.

\subsection{Sample Collection}

During visit, visual inspection was made to observe health status of the entire animals in the farms and detailed physical examination was done for sick animals suspected of having LSD. A total of 31 skin nodules were taken from representative sick animals for virus isolation and antigen detection according to the procedures of OIE (2010). The samples were taken aseptically by cleaning the area and removing the hairs with the help of sterile scalpel blade. Tissue samples were placed in the sterilized universal bottle and transported to NVI virology laboratory within 4 to 8 hours of collection within icebox and kept at $-20{ }^{\circ} \mathrm{C}$ until processed. 


\subsection{Laboratory Techniques}

\subsubsection{Sample Processing}

The skin biopsy samples were thawed at room temperature and washed three times using sterile phosphate buffer saline (PBS) at a pH of 7.2 in Bio-safety cabinet Class II. The biopsy tissue was minced using sterile scissors and forceps. About $1 \mathrm{gm}$ of the sample was ground using sterile pestle and mortar by adding $9 \mathrm{ml}$ of the sterile PBS at a $\mathrm{pH}$ of 7.2 containing $0.1 \%$ gentamycin. The tissue suspension was centrifuged at $1,500 \mathrm{rpm}$ for $15 \mathrm{~min}$. The supernatant was collected, filtered through $0.45 \mu \mathrm{m}$ pore size (Millipore, USA), labeled and preserved at $-20{ }^{0} \mathrm{C}$ until use.

\subsubsection{Isolation and identification of the virus}

The field isolated poxvirus was inoculated on Vero cell lines according to the method of Balinsky et al. (2008) to ascertain the presence of infectious virus. The Vero cell line was propagated in Glasgow Eagle minimal essential medium (GMEM) supplemented with 10\% calf serum. Vero cell line was grown in $25 \mathrm{~cm}^{2}$ tissue culture flask and was incubated with $5 \% \mathrm{CO}_{2}$ at $37^{\circ} \mathrm{C}$ until the cells became confluent monolayer. The tissue culture medium was then removed in aseptic conditions and the monolayer washed three times using sterile warm PBS pH of 7.2 in Bio-safety cabinet level II. One $\mathrm{ml}$ of tissue homogenate was inoculated onto the confluent monolayer and incubated at $37{ }^{\circ} \mathrm{C}$ and allowed to absorb for 1 hour. Then after covered the monolayer with $10 \mathrm{ml}$ of GMEM, containing antibiotics and $2 \%$ fetal calf serum into the flask and placed into incubator. All the flasks, including the control flasks, were incubated at $37^{\circ} \mathrm{C}$ in a humidified incubator with $5 \% \mathrm{CO} 2$. The medium was changed every 48 hours. Cells were monitored daily using an inverted microscope for evidence of virus induced cytopathic effects (CPEs) for 7- 14 days post-inoculation. Three more blind passages were carried out for samples that were initially CPE. Infected cells developed a characteristic CPE consisting of retraction of the cell membrane from surrounding cells; eventually rounding and aggregation of cells were observed. When $80 \% \mathrm{CPE}$ was observed, Virus inoculated flasks were harvested and frozen overnight at $-20^{\circ} \mathrm{C}$. The harvested cell culture was thawed twice at room temperature to release the virus particles. Finally, virus suspensions were stored at $-20^{\circ} \mathrm{C}$ until processed for viral DNA detection.

\subsubsection{DNA Extraction}

DNA extraction was performed using DNeasy Blood and tissue kit (Qiagen, Rocm and Haas Company, USA) according to the procedures of the manufacturer. DNA was extracted from 26 specimens; $180 \mu 1$ crude virus in culture supernatant from the LSDV infected cells were pipette in to $2 \mathrm{ml}$ of micro centrifuge tube, clarified by centrifugation at 8,000 rpm for $5 \mathrm{~min}$. $200 \mu \mathrm{l}$ of AL buffer (lysis buffer) was added in to the centrifuged virus suspension and mixed by vortexing and incubated at $56^{\circ} \mathrm{c}$ for $10 \mathrm{~min} .200 \mu \mathrm{l}$ of ethanol (100\%) was added and mixed thoroughly with the help of vortex mixer. The mixture was transferred in to DNeasy minispin column in $2 \mathrm{ml}$ collection tube and centrifuged at $8,000 \mathrm{rpm}$ for $5 \mathrm{~min}$. The spin column was transferred in to a new $2 \mathrm{ml}$ collection tube and $500 \mu \mathrm{l}$ of $\mathrm{AW}_{1}$ centrifuged at 8,000 rpm for $1 \mathrm{~min}$. Then, the collection tube was discarded and the min spin column placed in a new $2 \mathrm{ml}$ collection tube and $500 \mu \mathrm{l}$ of AW2 was added and centrifuged for $3 \mathrm{~min}$ at $14,000 \mathrm{rpm}$, min spin column transferred carefully in to a new $2 \mathrm{ml}$ of micro centrifuge tube and $200 \mu 1$ of elution buffer (AE buffer) added and incubated for $1 \mathrm{~min}$ at room temperature centrifuged for $1 \mathrm{~min}$ at 8,000 rpm. 2.5.4. Polymerase Chain Reaction (PCR)

A polymerase chain reaction (PCR) was carried out to detect the virus using Capripoxvirus-specific primer: Forward and reverse had the sequences (SpGpRNAPol-F) 5' TCTATGTCTTGATATGTGGTGGATAG-3' and (SpGpRNAPol-R) 5' AGTGATTAGGTGGTGTATTATTTTCC-3', respectively. DNA amplification was carried out in a final volume of $50 \mu \mathrm{l}$ of $10 \mathrm{X}$ PCR buffer, $1.5 \mu \mathrm{l}$ of $\mathrm{MgCl} 2(50 \mathrm{Mm}), 1 \mu 1$ of dNTP( $10 \mathrm{Mm}), 1 \mu \mathrm{l}$ of forward primer, $1 \mu \mathrm{l}$ of reverse primer, $1 \mu \mathrm{l}$ of DNA template, $0.51 \mu \mathrm{l}$ of Taq DNA polymerase and $39 \mu 1$ of nuclease free water. Finally the mix was incubated in a thermal cycler: first cycle: All PCR experiments performed in a PTC100 thermal cycler (MJ-Research, Inc., Waltham, Massachusetts, USA) using the following amplification program: initial denaturation at $95 \mathrm{oC}$ for $1 \mathrm{~min} ; 40$ cycles of denaturation at $95 \mathrm{oC}$ for $30 \mathrm{~s}$, annealing at $55 \mathrm{oC}$ for $30 \mathrm{~s}$ and elongation at $72 \mathrm{oC}$ for $1 \mathrm{~min}$. An additional elongation step was performed at $72 \mathrm{oC}$ for $5 \mathrm{~min}$ and the PCR products were stored at $4^{\circ} \mathrm{C}$ until analysis.

2.5.5. Agarose gel electrophoresis

The isolated DNA from cell culture was analyzed by agarose gel electrophoresis as described by ManganaVougiouka et al. (1999) with some modification to confirm the presence of DNA. Amplified products were analyzed using a gen ruler ${ }^{\mathrm{TM}} 100 \mathrm{bp}$ DNA ladder (Fermentes, Germany) as a molecular marker on $3 \%$ agarose gels prepared in Tris/Acetate/EDTA (TAE) buffer and $10 \mathrm{mg} / \mathrm{ml}$ ETDM- bromide stain, then $20 \mu \mathrm{l}$ of PCR product was mixed with $4 \mu 1$ loading buffer and loaded to wells in previously prepared gel and run at 100 volt for about 60 minutes in parallel with DNA molecular weight marker in electrophoresis apparatus until the DNA samples have migrated a sufficient distance through the gel. DNA bands were visualized using UV transilluminator at a wave length of $590 \mathrm{~nm}$, and positive results were confirmed according to the size of the bands formed on agarose gel. The PCR results were considered positive for LSDV and GTPV DNA when a 172 bp and SPPV 151 bp product were observed. 


\subsubsection{Real-time PCR}

The real-time PCR was executed at Molecular Biology Laboratory, NVI. Each sample was tested in duplicate and every PCR run included no-template and positive GTPV, LSDV and SPPV controls. 5'GGTGTAGTACGTATAAGATTATCGTATATA-3' ' as forward primer and 5'AATTTCTTTCTCTGTTCCATTTG-3' as reverse primer were used for Real-time PCR analysis.

The PCR was set up in a $20 \mu \mathrm{L}$ reaction volume. A total of $1430 \mu 1$ of the master mix was prepared to 26 samples. $17 \mu \mathrm{l}$ of the master mix was dispensed into each micro-well of Low Profile Hard-Shell H 96-well PCR plate (BioRad). The master mix in each well was composed of $4.84 \mu \mathrm{l}$ of RNAase free water, $2 \mu 1$ of forward primer (CHFRsb), $0.16 \mu \mathrm{L}$ of reverse primer (CHFR1), and $10 \mu \mathrm{L}$ of 1x SsoFast Eva Green Super mix. $3 \mu \mathrm{L}$ of sample extracted DNA; no-template (RNAase free water); and positive GTPV, LSDV and SPPV controls was added in duplicate fashion and sealed. PCR was performed in a CFX $96^{\mathrm{TM}}$ real-time PCR detection system with an initial denaturation step at $95{ }^{\circ} \mathrm{C}$ for 3 minutes, followed by 45 cycles of $95{ }^{\circ} \mathrm{C}$ for 15 seconds and $58{ }^{0} \mathrm{C}$ for 80 second using a Low Profile Hard-Shell H 96-well PCR plate (Bio-Rad). The product was then denatured at $95{ }^{\circ} \mathrm{C}$ (held for 1 minute), cooled to $40^{\circ} \mathrm{C}$ (held for 1 minute), and heated continuously at $0.5^{\circ} \mathrm{C} / 10$ seconds with fluorescence acquisition from $40{ }^{\circ} \mathrm{C}$ to $85{ }^{\circ} \mathrm{C}$. The melting temperatures were analyzed using the $\mathrm{CFX}^{\mathrm{TM}}$ Manager Software Version 2.0 (Bio-Rad) and the correspondent curves were displayed as negative first-derivative plots of fluorescence with respect to temperature. High-Resolution Melting (HRM) analysis, a post-PCR melting analysis method used to identify variations in nucleic acid sequences, was also used to plot the melting profile of the three genotypes using the Precision Melt Analysis ${ }^{\text {TM }}$ Software (Bio-Rad). Normalized melt curves and difference in curves were acquired by selecting pre-and post-melt regions for amplicons separately (Lamien et al., 2011b).

2.5.7. RPO30 gene sequencing

The RPO30 gene sequence was done for only seven PCR positives representative samples in the study areas. The DNA extracts were sent to Vienna, Austria, for gene sequencing. The PCR was also conducted to amplify the DNAs from the extracts. PCR products were also checked using electrophoresis on a 1.5\% agarose gel for $1 \mathrm{~h}$ at $100 \mathrm{~V}$. The positive DNA products were purified using Wizard SV Gel and PCR clean-up system kit (Promega) and sequenced commercially by LGC Genomics (Germany).

\subsection{Data Analysis}

The data obtained from all investigations were coded and stored in Excel spread sheet 2007 and thoroughly screened before subjected to statistical analysis. Statistical analysis was applied using SPSS statistical software version 19.0. Morbidity and mortality were estimated in accordance with vaccination history, farm types, and age categories (in big dairy farms). Mean and Confidence Interval (CI) were also used to describe morbidity and mortality across different variables. The effectiveness of the Current LSD vaccine was analyzed in the existing vaccination strategies. Field vaccine effectiveness was assessed by the percentage reduction in risk of having LSD among regularly vaccinated dairy cattle compared to dairy cattle vaccinated during outbreaks. The formula used for calculating vaccine effectiveness (VE) in this study was VE $=[(\mathrm{RIV}-\mathrm{RRV}) / \mathrm{RIV}] * 100 \%$, where RIV is risk of irregular vaccination (risk of having LSD in dairy cattle vaccinated during the onset of outbreak and RRV is relative risk of vaccinated group before outbreak onset. Chi-square $\left(\chi^{2}\right)$ was employed to test the presence of association among different categorized variables. In all the analyses, confidence levels at $95 \%$ were calculated, and a $\mathrm{P} \leq 0.05$ was used for statistical significance level (Moulton et al., 1995).

For genomic analysis, the sequence data were edited and fragments were assembled using Vector NTI Advance $^{\mathrm{TM}} 11.5$ software (Invitrogen, Carlsbad, CA, USA). Phylogenetic tree analysis was included 35 capripoxviruses based on nucleotide sequences of RPO30 gene complete sequence (606bp). LSDV field isolates, attenuated vaccine strain (KS-1 and Gorgon) and reference sequences retrieved from the Genbank database were considered in the analysis. The Neighbor-Joining method with the maximum composite likelihood nucleotide substitution with the pairwise deletion option was computed using the Kimura 2-parameter method of MEGA6 software (Tamura, 2013). The percentages of bootstrap scores above 50\% (out of 1000 replicates) are shown next to the branches.

\section{RESULTS}

\subsection{Observed Clinical Signs}

The common clinical signs observed in our study were fever, circumscribed nodules on the skin with different sizes, necrotic nodules and deep scab formation, edematous swelling of one or two legs, enlargement of superficial lymph nodes and decrease in body weight (Fig 1). Lameness and superficial lymph node enlargement were very prominent. The nodules on the limbs gradually burst and left necrotic wounds that were frequently seen to be complicated with secondary infection (Fig.2). Dysentery and recombency were also seen. 


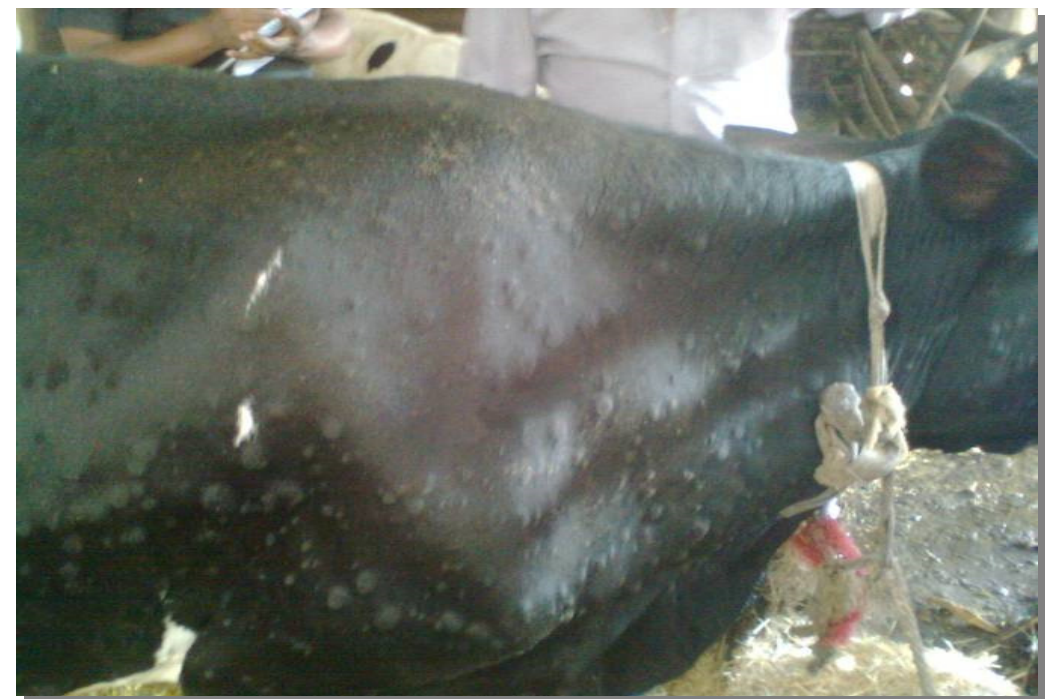

Figure 1: Characteristic of LSD with generalized circumscribed skin nodules covering the entire body

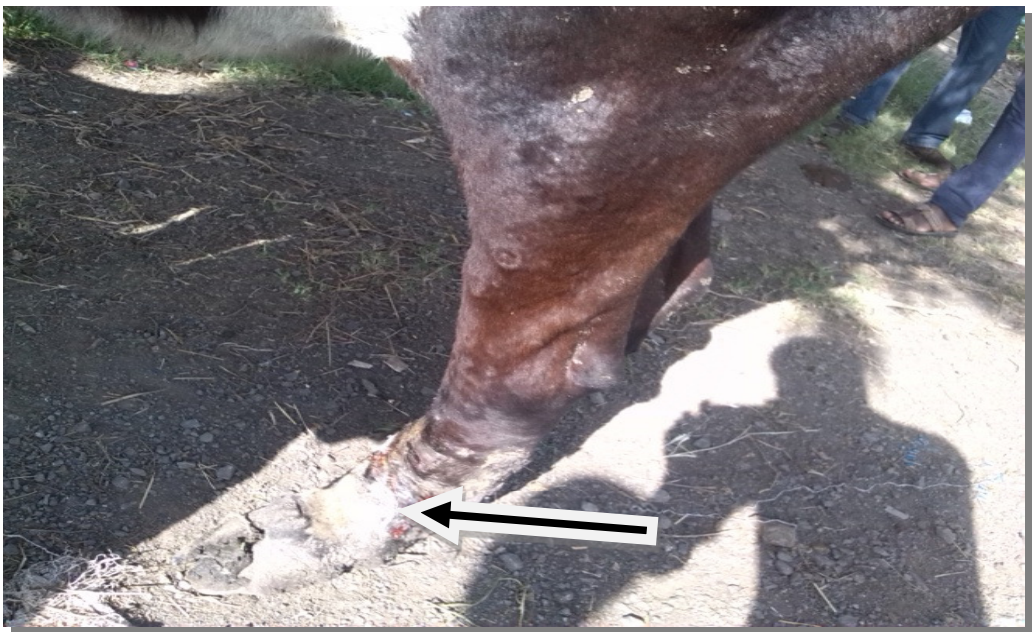

Figure 2: An inflammatory swelling of the hind leg following complicated wound.

\subsection{Virus Isolation}

Out of the 31 skin biopsies, characteristic poxvirus CPE was observed in infected Vero cells with 26 samples within ten days of post- inoculation or after one or two blind passages while the virus was not isolated from the remaining five samples. None of the negative control cultures showed any CPE after two or three blind passages.
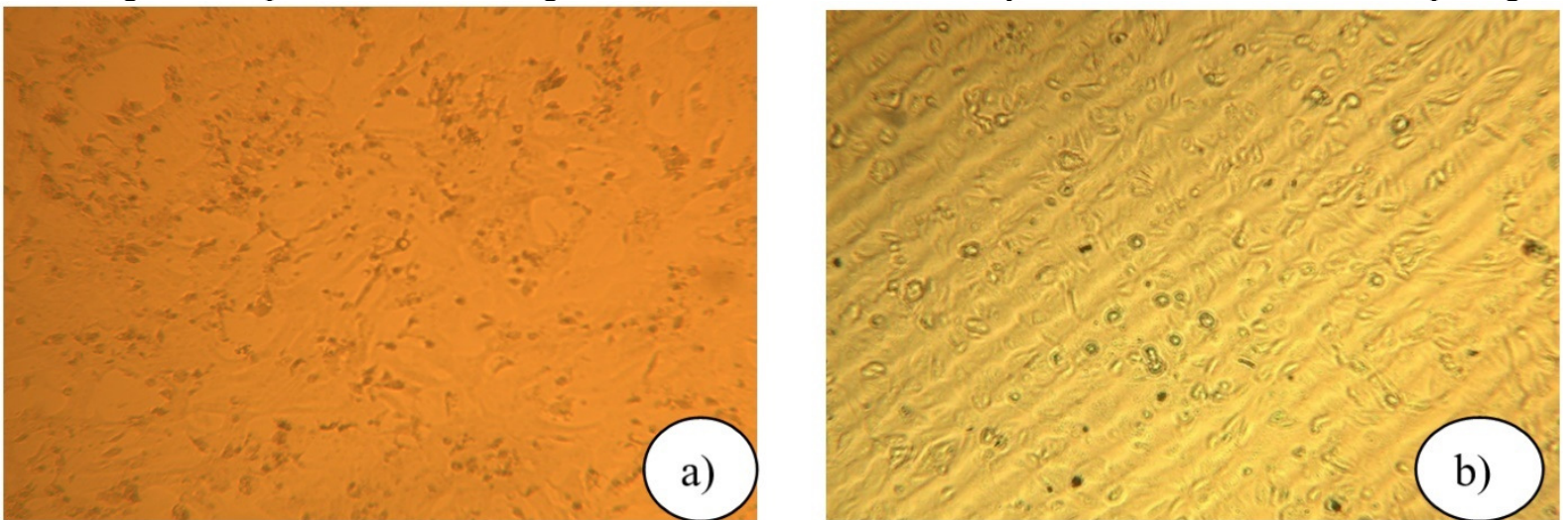

Figure 3: Photo of Vero cell: a) CPE positive on Vero cell after seven days post inoculation; b) control of cell culture

\subsection{Polymerase Chain Reaction}

The DNAs of 26 isolates on Vero cell line were amplified using capripoxvirus-specific primers indicated in section 
3.5.4. The amplicon size of PCR product had molecular weight of $172 \mathrm{bp}$ (Fig.7), the expected amplicon size for the LSDV genomic region targeted. The resulting PCR products of GTPV/LSDV differed in length by 21 nucleotides produced from SPPV genomes. After electrophoresis migration of the PCR products on a 3\% highresolution agarose gel, all amplicons from the SPPV group were shorter (151 bp) and easily distinguishable, relative to the GTPV/LSDV amplicons 172 bp (Fig. 7).

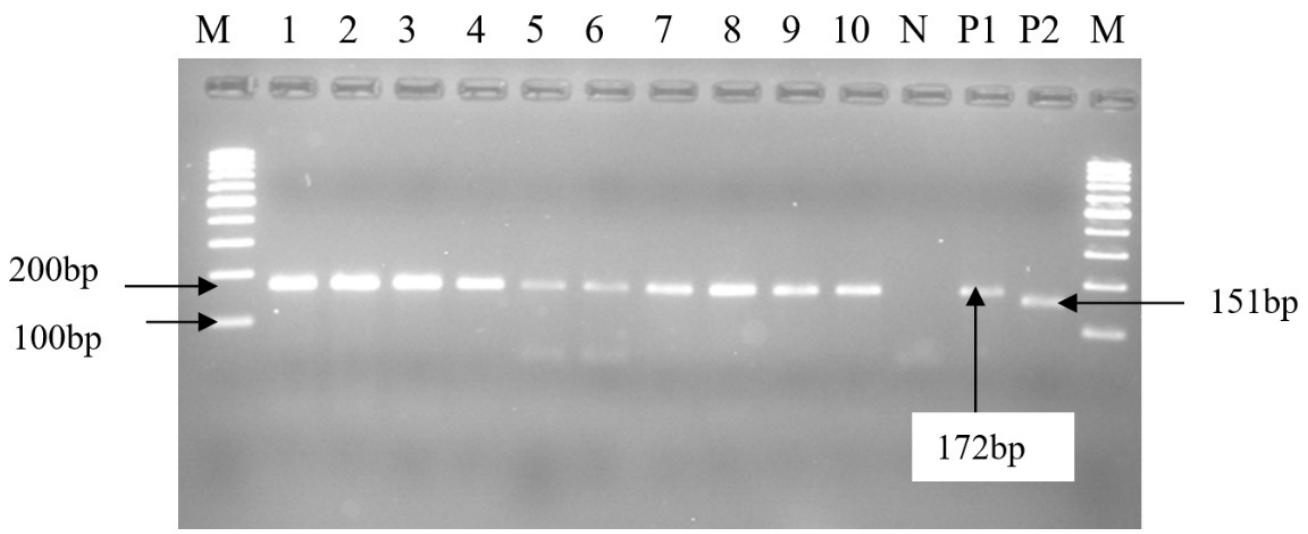

Figure 4: PCR based detection of LSDV in samples taken from skin nodules of infected animals.

Lanes M: DNA ladder; lane N: Negative Control without template; Lanes 1, 2 and 3 represent positive samples from Bishoftu; Lanes 4 and 5 represent positive samples from Holeta; Lanes 6 and 7 represent positive sample from Ginchi; Lane 8 and 9 is a positive sample from Selale; Lane 10 is a positive sample from Akaki; $\mathrm{P}_{1}$ - Positive control for LSD and Goat pox- (size 172bp); P2- Positive control for Sheep pox- (size 151bp).

\subsection{Real-time PCR}

The DNA extracts forming 172 bands on agarose gel was again subjected to real-time PCR and LSDV differentiated from GTPV. The peak melting curve of the real time revealed that all 26 virus isolates were characterized as LSDV since their snapback of melting peaks were at $51{ }^{\circ} \mathrm{C}$ while the second peaks were at $73.5^{\circ} \mathrm{C}$ (Fig 8 ). Known CaPV positive samples were tasted for comparison. Real-time PCR assay detected differences in the melting point temperatures for SPPV, GTPV and LSDV after fluorescence melting curve analysis from each other (Fig 9).

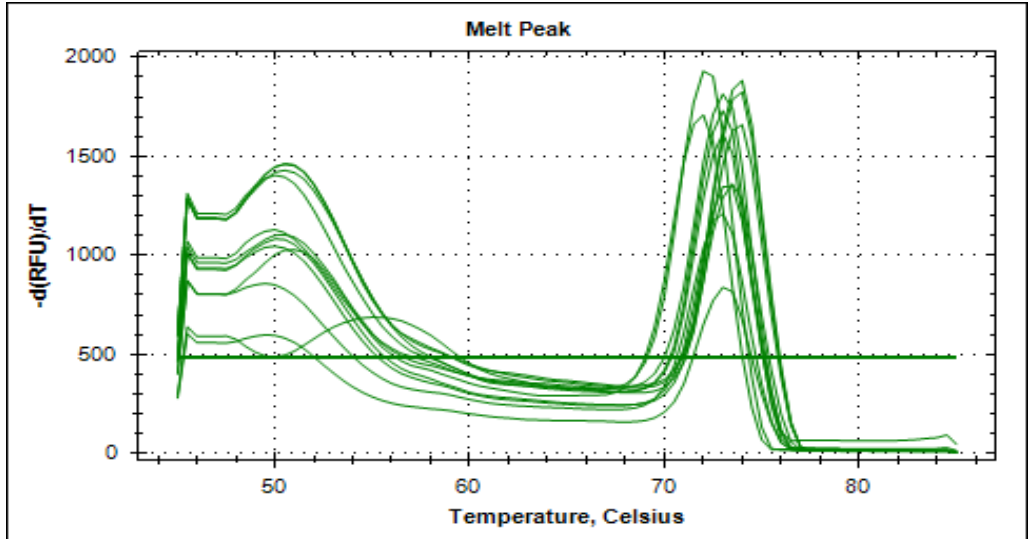

Figure 5: Differences in the melting curve for field isolates of LSDVs 


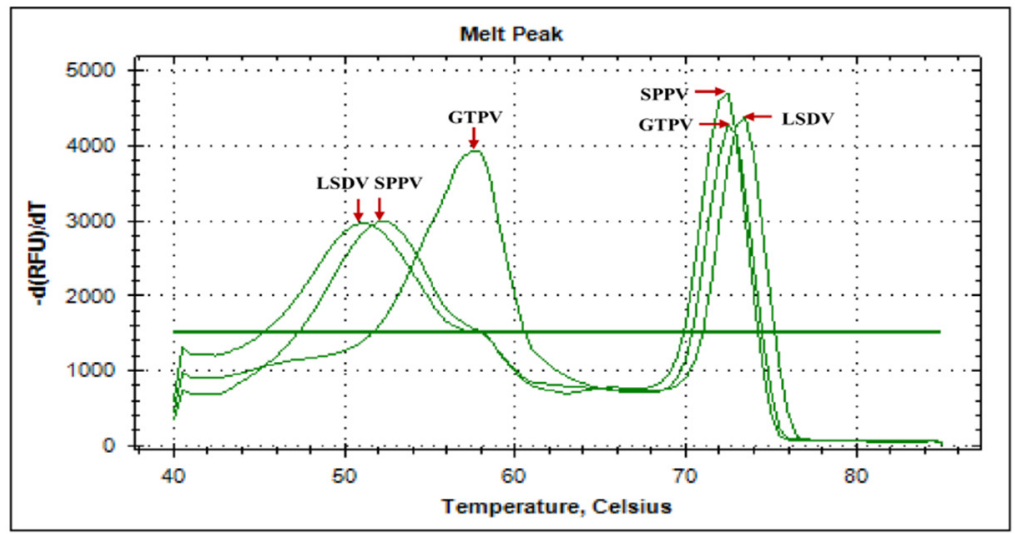

Figure 6: Differences in the melting curve for known positive isolates of CaPVs for comparison.

The RPO30 genes sequence analysis of the current Ethiopian field isolates and the previous LSDV isolates showed that substitution of some nucleotides (1-5) with respect to the current NVI vaccine strain (KS-1). $\begin{array}{lllllll}10 & 20 & 30 & 40 & 270 & 280 & 290\end{array}$

NVI/CaPV Vaccine (KS-1) KP66368 ATGGATGATGATAATACTAATCCATATAGIGATAATACDACCCQGATAGAGACAAATAAAGATTATCCTGI EDLIT/2014

Kaliti/2014

Ambo / 2014

Ginchi /2014

Selale/2014

Holeta/2014

Quarit

Wenji/B03/2011 KP663689.1

Mojo/B01/2011 KP663683.1

Debre zeit/B01/2009 KP663680.1

Sudan/99 Atbara Gr119944.1

Kenyan SGP 0-240 kJ818288.1

Adama/B01/2011 KP663667.1

Andassa/B05/2012 KP663672.1

Goatpox Metekel/001/2010 KP663



Figure 7: Multiple nucleotide sequence alignment of RPO30 gene of present and previous Ethiopian LSDV field isolates, NVI vaccine strain (KS-1), Kenyan SGP O-240 and Ethiopian field GTPV. Sequence analysis of the Ethiopian field LSDV isolates showed substitution of at least one nucleotide as compared to the vaccine strain (KS-1).

The $30 \mathrm{KDa}$ DNA-dependent RNA polymerase subunit (RPO30) genes (Lamien et al., 2011a) of capripoxvirus were analyzed in order to characterize and compare local isolates and the local vaccine strain. Based on multiple sequence alignment of the present isolates' RPO30 gene complete sequences of 606 bp with KS-1 vaccine, all the present isolates, C nucleotide substituted by T (at nucleotide position 292) while Ambo, Ginchi, Holeta, Quarit and Selale isolates differed by additional one nucleotide substitution (at nucleotide position 41, C substituted by A) from KS-1 vaccine strain. These isolates were similar to previous isolate from East Shoa Zone of Oromia Regional State, Ethiopia.

\subsection{Phylogenetic Analysis}

Finally, phylogenetic reconstructions were performed to determine the genetic relationship among Ethiopian isolates as well as other $\mathrm{CaPV}$ isolates. In general, phylogenetic analysis indicates that all representative isolates $(\mathrm{n}=7)$ were identical to LSDV and related to SPPV and GTPV. Phylogenic tree analysis categorized the present field virulent isolates into two clusters. The isolates obtained from Bishoftu and Kaliti were found to be $99 \%$ similar to the previously isolated LSDV strains from Adama, Andassa, Bishoftu, Modjo and Wenji. These isolates were also closely related to the isolates of Burkina Faso, Egypt and Niger. However, the isolates from Ambo, Ginchi, Holeta, Quarit (West Gojam) and Selale separately formed other cluster which was closely analogous to Sudan isolates (Fig 11). 


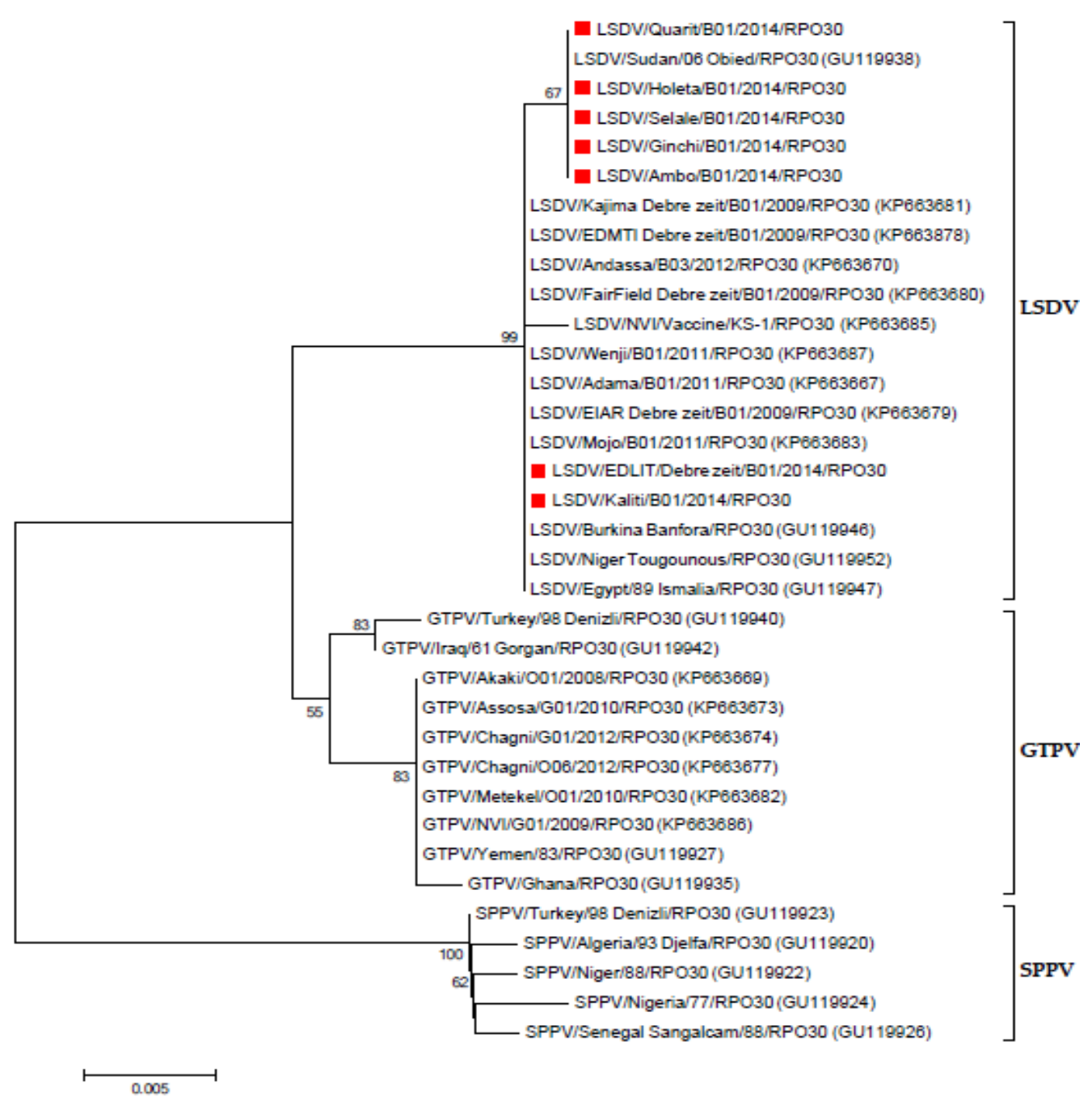

Figure 8: Phylogenetic tree analysis of 35 capripoxviruses based on nucleotide sequences of RPO30 gene complete sequence (606bp). The current isolates are marked with black square box.

\subsection{Vaccination Strategy against LSD}

The questionnaire survey revealed that, vaccination against LSD is usually done during outbreak eruptions in the areas. All visited smallholder dairy farm owners responded that they did not have regular vaccination program against the disease. Out of 17 smallholder dairy farms, three owners of the Akaki-Kaliti sub-city of Addis Ababa and three owners of Bishoftu town gave the vaccine after they had already observed disease in their farms. However, the remaining 11 owners $(64.7 \%)$ vaccinated their animals prior to the occurrence of the first case in their farms. In such vaccination strategy, drastic increments of the number of new cases were a big concern for the farm owners and animal health workers. In contrary, big dairy farms do have their own regular vaccination schedule and vaccinate their animal annually against LSD before the beginning of the main rainy season. Despite their efforts, LSD outbreak was seen in assessed big dairy farms after six to ten months of the last vaccination (Annex 3). Isolation of infected animals and broad spectrum treatment such as $20 \%$ Oxy tetracycline and penstrips were the intervention measures taken by all big dairy farms.

\subsection{Outbreak Investigation and LSD Vaccine Effectiveness}

Out of 1742 dairy cattle observed, 213 animals (12.2\%) were developed LSD clinical signs with physical examination. Low morbidity rate of $10 \%(95 \% \mathrm{CI}=0.08-0.11)$ was recorded in regularly (annually) vaccinated cattle, whereas high morbidity of $30.4 \%(95 \% \mathrm{CI}=0.24-0.37)$ was found in dairy cattle vaccinated during outbreaks. The $95 \%$ CIs of morbidity rates elicits that regularly vaccinated dairy cattle and dairy cattle vaccinated during outbreak were statically different. LSD vaccination strategy was also significantly associated $\left(\mathrm{P}<0.05, \chi^{2}=\right.$ 65.768 ) with the morbidity rate (Table 2 ). 
Table 1: Morbidity of LSD in dairy cattle having regular vaccination and vaccination during outbreak

\begin{tabular}{|l|l|l|l|l|l|l|}
\hline Regular vaccination & Total animals & No. of sick animals & Morbidity & $\mathbf{9 5 \%}$ CI & $\boldsymbol{\chi}^{\mathbf{2}}$ & P-value \\
\hline Present & 1551 & 155 & 0.100 & $0.08-0.11$ & 65.768 & 0.000 \\
\hline Absent & 191 & 58 & 0.304 & $0.24-0.37$ & & \\
\hline Total & $\mathbf{1 7 4 2}$ & $\mathbf{2 1 3}$ & $\mathbf{0 . 1 2 2}$ & $\mathbf{0 . 1 1}-\mathbf{0 . 1 4}$ & & \\
\hline
\end{tabular}

On the other hand, only 27 dairy cattle $(1.5 \%)$ were dead from all visited dairy animals. Mortality rate of $0.6 \%$ $(95 \% \mathrm{CI}=0.00-0.01)$ in regularly vaccinated dairy cattle was lower than mortality of dairy cattle vaccinated during outbreak $9.4 \%(95 \% \mathrm{CI}=0.05-0.14)$. The $95 \%$ CIs of the groups illustrate absence of statistical difference between the two dairy cattle populations. Statistical significant difference $\left(\chi^{2}=87.165, P<0.05\right)$ was revealed between LSD mortality rate and vaccination history.

Table 2: Mortality of LSD in dairy cattle with different vaccination history

\begin{tabular}{|l|l|l|l|l|l|l|}
\hline Regular vaccination & Total animals & No. of dead animals & Mortality & $\mathbf{9 5 \%}$ CI & $\boldsymbol{\chi}^{\mathbf{2}}$ & P-value \\
\hline Present & 1551 & 9 & 0.006 & $0.00-0.01$ & 87.165 & 0.000 \\
\hline Absent & 191 & 18 & 0.094 & $0.05-0.14$ & & \\
\hline Total & $\mathbf{1 7 4 2}$ & $\mathbf{2 7}$ & $\mathbf{0 . 0 1 5}$ & $\mathbf{0 . 0 1 - \mathbf { 0 . 0 2 }}$ & & \\
\hline
\end{tabular}

In the context of the existing vaccination strategy, the relative risk of having LSD in annually vaccinated dairy cattle was $32.9 \%(95 \% \mathrm{CI}=0.253-0.427)$ lower than in dairy cattle vaccinated during outbreaks. Therefore, the effectiveness of regular annually vaccination against LSD was estimated to be $67.1 \%(95 \% \mathrm{CI}=57.3 \%-74.7 \%)$ as compared to vaccination during outbreak occurrences. The relative risk of death in regularly vaccinated dairy cattle was calculated to be $6.2 \%(95 \% \mathrm{CI}=0.028-0.135)$ and thus, $93.8 \%$ mortality reduction was observed in regularly vaccinated dairy cattle.

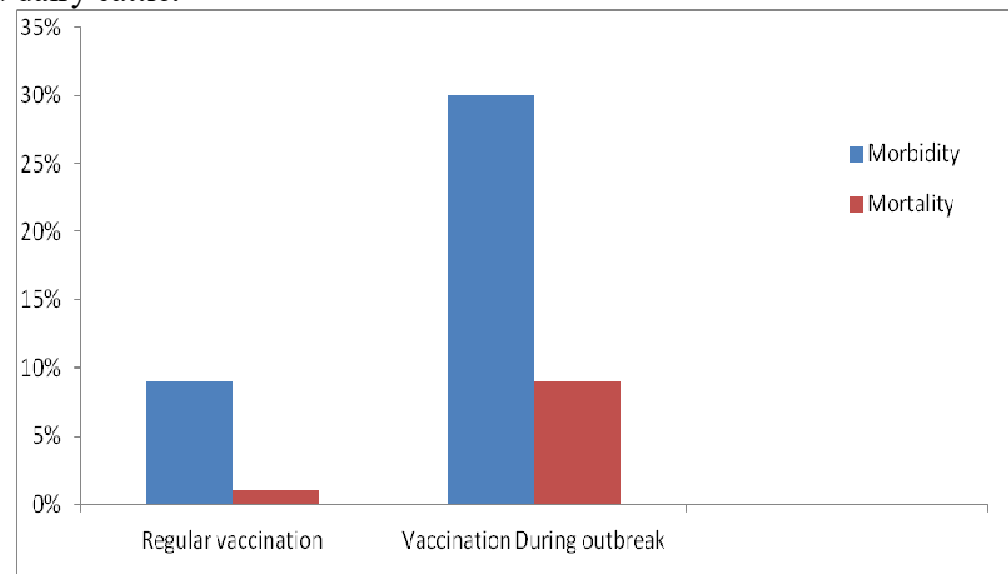

Figure 9: Morbidity and mortality rates in dairy cattle with different vaccination strategy

Morbidity was done in three big dairy farms (B-EIAR not considered) and the study included a total of 1436 cattle. Affected animals in regular annual vaccinating farms were divided into two age groups: young $(<2$ years) and adult ( $\geq 2$ years). There was higher morbidity of $31 \%(95 \% \mathrm{CI}=0.26-0.36)$ observed in young cattle group than $3 \%(95 \% \mathrm{CI}=0.02-0.04)$ morbidity rate in the adults. This difference was highly significant since the two groups of $95 \%$ CIs didn't overlapped. The age specific morbidity was statistically significant association $\left(\chi^{2}=\right.$ $2.434, P<0.05)$ to their age group in big dairy cattle.

Table 3: Morbidity of LSD in regularly vaccinated three big dairy cattle by age category

\begin{tabular}{|l|l|l|l|l|l|l|}
\hline Age category & Total animals & No. of sick animals & Morbidity & $\mathbf{9 5 \%}$ CI & $\boldsymbol{\chi}^{\mathbf{2}}$ & P-value \\
\hline$<2$ years & 389 & 121 & 0.31 & $0.26-0.36$ & 2.434 & 0.000 \\
\hline$\geq 2$ years & 1047 & 29 & 0.03 & $0.24-0.04$ & & \\
\hline Total & $\mathbf{1 4 3 6}$ & $\mathbf{1 5 0}$ & $\mathbf{0 . 1 0}$ & $\mathbf{0 . 0 9 - - 0 . 1 2}$ & & \\
\hline
\end{tabular}

\section{DISCUSSION}

The findings of clinical signs, virus isolation, and PCR diagnosis confirmed that the outbreak was caused by LSDV. Clinical signs observed on LSD infected dairy cattle were fever, circumscribed skin nodules, enlargement of lymph nodes, inappetence, lacrymation, salivation, reduction of milk production and death. These signs have been documented as characteristic clinical features of LSD (OIE, 2012; Radostits et al., 2007; Carn and Kitching, 1995). Edematous swelling of legs and lameness were very common signs. Similar situation was recorded during Oman LSD outbreak (Mohamed et al., 2014). The complications such as dysentery and recombency were also reported from Egypt and Oman (Mohamed et al., 2014). Out of 31skin biopsies, characteristic of Capripoxvirus CPE was observed only on 26 skin samples following post-inoculation on Vero cell line. Whereas, virus isolates could not 
be identified from the remaining five skin samples. This might show the absence of infectious virus particles from scarified skin tissue. All isolates were identified as LSDV after sequential diagnosis using convention PCR and real-time PCR.

Furthermore, molecular phylogenetic analysis confirmed the identifications made using real-time PCR. Multiple alignment analysis of complete sequences of RPO30 gene $606 \mathrm{bp}$ of present field virulent isolates, KS-1 vaccine strain and past isolates revealed that there was high homology percentage (99\%) of the nucleotide sequence among the current Bishoftu and Kaliti isolates and previous isolates from Adama, Andassa, Bishoftu, Modjo and Wenji. This result rules out that these virulent LSDV field strains are still circulating in the eastern central part of the country. Other present isolates (Ambo, Ginchi, Holeta, Quarit and Selale) were slightly different from the former isolates by replacing A nucleotide by $\mathrm{C}$ (nucleotide position 41) and formed a separate cluster which is related to Sudan strain. Interestingly, all the current and previous field isolates have substituted $\mathrm{T}$ nucleotide by $\mathrm{C}$ (nucleotide position 292) in contrast to vaccine strains. Even though these findings might not show any change in amino acid substitutions, Gelaye et al. (2015) unveiled 12 nucleotides deletion from the previous isolates of GPCR genes as compared to KS-1 vaccine strain.

Attenuated KS-1 vaccine strain has been produced by NVI and distributed across the country to control LSD. The vaccine is usually used for stopping ongoing outbreaks by mass vaccination campaign, except in big dairy farms for annual vaccination. This vaccine strain was thought to be SPPV in the past years, but it has confirmed to be LSDV in recent years (Gelaye et al., 2015; Tuppurainen et al., 2014). The Yugoslavian RM 65 sheep-pox virus strain, the Romanian sheep-pox virus strain and the South African Onderstepoort LSDV strain are recommended vaccines to control LSD (Kitching, 2003; Carn and Kitching, 1995, OIE, 2012). All these strains of capripoxvirus were predicted that they could protect cattle against LSDV as they share a major neutralizing epitope. Moreover, it is claimed that vaccinated animals will develop protective immunity from 10 to 21 days postvaccination, and then required an annual booster dose (OIE, 2010).

In contrary, outbreaks were appeared in vaccinated herds after five to ten months of the last vaccination date and two weeks to two months of post vaccination in smallholder dairy farms. The incomplete protection following vaccination discouraged dairy farmers to vaccinate their animals, consequently rendering more dairy cattle at risk. Abutarbush (2014) has also reported the occurrence of outbreak after 5 to 20 days post vaccination. Postvaccination LSD outbreak occurrence may be indicative of incomplete protection. This finding is in support of incomplete protection of KS-1 vaccine strains against circulating virulent field strains in Ethiopia (Ayelet et al., 2014) and in Oman (Mohamed et al., 2014). Such situation of other vaccine strains has been reported in Egypt and Israel (Brenner et al., 2009), and Jordan (Abutarbush, 2014). Furthermore, there was increasing new cases following mass vaccination during outbreak eruptions. This might be result of vaccination of incubatory cattle accompanied by unhygienic vaccination.

In the present study, morbidity $(12.2 \%)$ and mortality $(1.5 \%)$ were recorded in the dairy cattle regardless of vaccination history. These findings are in agreement with $13.5 \%$ of morbidity rate (Brenner et al., 2009) and low mortality rates $1.26 \%$ (Gari et al., 2011). However, several literatures reported that the morbidity and mortality rates of LSD vary. The morbidity rate of the disease ranges from 5 to $100 \%$ (Woods, 1988). While occasional mortality rates from 10 to $40 \%$ have been reported (EMPRES, 2013), but the rate of 1 to $5 \%$ is usually observed (Woods, 1988). These values vary with geography, climate, management conditions, and immune status of the animals, breed and strain of virus involved (Tuppurainen and Oura, 2012).

In the present study, the morbidity in dairy cattle vaccinated during outbreak was more than three times with that of annually vaccinated dairy cattle. The morbidity in annually vaccinated herds $(10 \%)$ is in agreement with morbidity rate of $9.5 \%$ (Abutarbush, 2014) and 11.1\% (Brenner et al., 2009) in vaccinated cattle population. A morbidity rate of $30.4 \%$ in dairy farms vaccinated after outbreak eruption is in comparable with the report of $22.5 \%$ morbidity in cattle vaccinated after infection (Abutarbush, 2014).

On the other hand, the present mortality in dairy cattle vaccinated during outbreaks was recorded to be more than 15 times higher than those of dairy cattle annually vaccinated. The mortality of $0.6 \%$ in regularly vaccinated dairy cattle is relatively lower than $2.4 \%$ morality observed vaccinated Jordanian cattle (Abutarbush, 2014). Two third of dead dairy cattle as result of the LSD outbreak were reported in lactating and pregnant cows. Stressful physiological status of the animals makes them prone to the disease. The average price of a cow during study period was estimated to 35,000 Eth birr while 25,000 Eth Birr for a heifer. Thus, although economic losses mostly from morbidity (Woods, 1988), a mortality of $9.4 \%$ causes a heaviest economical burden on poor smallholder dairy farm owners whose livelihood mainly relies on.

Attempt was also made to compare KS-1 vaccine effectiveness between the existing vaccination strategies. In the present study, the relative risk of having LSD infection in regularly vaccinated dairy cattle was 0.329 times lowering than the risk of vaccinated during outbreaks. Thus, the vaccine effectiveness using annual vaccination was estimated to be $67.1 \%$ as compared to vaccination when outbreak occurrences. On the other hand, the implementation of annual vaccination in smallholder dairy farms could also reduce their mortality by $93.8 \%$. Most of cattle used for dairy purpose were cross bred with Holstein-Friesian breed which are relatively susceptible to 
LSD infection (Babiuk et al., 2008). Therefore, annual vaccination using KS-1 vaccine is still the existing alternative to reduce the impact of the disease in susceptible diary animals. However, the observation of a morbidity of $10 \%$ in annually vaccinated dairy cattle is frustrating and urges the development of better, effective and safe LSD vaccine.

In regularly vaccinated dairy cattle, morbidity of young animals were ten times higher than adult cattle. This age specific susceptibility is in accordance to the findings of Ayelet et al. (2014). Calves less than six months of age were not received the vaccine during vaccination and the number of calves exhausting their maternal immunity increases as the last vaccination date is advancing. Conversely, adult herd could build strong immunity against infection due to repeated vaccination or natural infection. Thus, herd immunity variation possibly explains morbidity difference between the two age categories in the present study.

\section{CONCLUSIONS AND RECOMMENDATIONS}

The present outbreak investigated in dairy cattle was caused by LSDV. Smallholder dairy farms were highly affected by the outbreak as compared to big dairy farms. The presence of regular vaccination in big dairy farms was significantly associated to the observed relatively low morbidity and mortality rates. Despite vaccination during outbreak eruption, increasing mortality of the outbreak causes economic burden to poor smallholder dairy farm owners whose livelihood is heavily relied on dairy farming. It is undeniable that LSD outbreak was occurred in vaccinated dairy cattle. Such phenomenon is an indicative of KS-1 vaccine incomplete protection. The current study shows that there is nucleotide missing among the circulating isolates of LSDV in relation to the vaccine strain (KS-1). Recently this event could be another challenge for the effective control of the LSD. This poor protection of the vaccine can discourage the dairy farmers from using vaccination to protect their herds, thereby putting more animals at risk. However, annual vaccination against LSD using this vaccine provides a better protection to dairy cattle with respect to dairy cattle vaccinated during outbreak eruptions. Younger animals were more susceptible age group in regular vaccinated dairy cattle herds. Based on the above conclusion the following recommendations are forwarded.

- Regular annual vaccination should be implemented in every dairy farm since it is still the only alternative for LSD susceptible animals in endemic countries;

- Efforts need to be directed for the development of an effective vaccine, thereby contributing to better control of LSD in the future;

- Detailed molecular studies should be done to discover the variation of circulating virulent field strains, thus developing new vaccines by targeting genes specifically involved in virulence and host immune system modulation.

\section{Acknowledgments}

The authors would like to thank National Veterinary Institute (NVI) for providing us the fund and facilities required to conduct the entire work. We would also like to thank Mr. Alebachew Belay and Dr. Teferi Degefa for their technical assistance.

\section{Ethical Statement}

This research was approved for study by the animal research ethics committee of college of Veterinary Medicine and Agriculture, AAU.

\section{Conflict of interest}

The authors declare that there is no conflict of interest regarding the publication of this article.

\section{REFERENCES}

1. Abutarbush, S.M., 2014. Efficacy of vaccination against lumpy skin disease in Jordanian cattle. Veterinary Record, 175, 302-305.

2. Abutarbush, S.M., Ababneh, M.M., Al Zoubi I.G., Al Sheyab, O.M., Al Zoubi M.G., Alekish, M.O., Al Gharabat, R.J., 2013. Lumpy skin disease in Jordan: disease emergence, clinical signs, complications and preliminary-associated economic losses. Transboundary and Emerging Diseases, 62, 549-54.

3. Ayelet, G., Abate, Y., Sisay, T., Nigussie, H., Gelaye, E., Jemberie, S., Asmare, K., 2013. Lumpy skin disease: preliminary vaccine efficacy assessment and overview on outbreak impact in dairy cattle at Debre-zeit, central Ethiopia. Antiviral Research, 98, 261-265.

4. Ayelet, G., Haftu, R., Jemberie, S., Belay, A., Gelaye, E., Sibhat, B., Skjerve, E., Asmare, K., 2014. Lumpy skin disease in cattle in central Ethiopia: outbreak investigation and isolation and molecular detection of the virus. Scientific and Technical Review of Office International Epizootics, 33, 877-87.

5. Babiuk, S., Bowden, T., Boyle, D., Wallace, D., Kitching, R., 2008a. Capri pox viruses: An emerging worldwide threat to sheep goats and cattle. Transboundary and Emerging Diseases, 55, 263-272. 
6. Balinsky, C.A., Delhon, G., Smoliga, G., Prarat, M., French, R.A., Geary, S.J., Rock D.L., Rodriguez, L.L, 2008. Rapid preclinical detection of sheeppox virus by a real-time PCR assay. Journal of Clinical Microbiology, 46, 438-442.

7. Brenner, J., Bellaiche, M., Gross, E., Elad, D., Oved, Z., Haimovitz, M., Wasserman, A., Friedgut, O., Stram, Y., Bumbarov, V., Yadin, H., 2009. Appearance of skin lesions in cattle population vaccinated against lumpy skin disease: statutory challenge. Vaccine, 27, 1500-1503.

8. Carn, V., M. and Kitching, R., 1995b. An investigation of possible routes of transmission of lumpy skin disease virus (Neethling). Epidemiology and Infection, 114, 219-226.

9. Carn, V.M., Kitching, R. P., 1995a. The clinical response of cattle following experimentally infected with lumpy skin disease (Neethling) virus. Archives of Virology, 140, 503-513.

10. Chihota, C., Rennie, L.S., Kitching, R.P., Mellor, P.S., 2001. Mechanical transmission of lumpy skin disease virus by Aedes aegypti (Diptera: Culicidae). Epidemiology and Infection, 126, 317-321.

11. Chihota, C.M., Rennie, L.F., Kitching, R.P., Mellor, P.S., 2003. Attempted mechanical transmission of lumpy skin disease virus by biting insects. Medical Veterinary Entomol, 17, 294-300.

12. Davies, F.G., Otema, C., 1981. Relationships of capripox viruses found in Kenya with two Middle Eastern strains and some orthopox viruses. Research in Veterinary Science, 31, 253-255.

13. Emergency Prevention System for Animal Health (EMPRES)., 2013. Emergence of lumpy skin disease in the Eastern Mediterranean Basin countries. In EMPRES watch, Volume 29. FAO of the UN; FAO Regional Office for the Near East.

14. Gari,G., Waret-Szkuta, A., Roger, F., Bonnet, P., 2011. Epidemiological aspects and financial impact of lumpy skin disease in Ethiopia. Preventive Veterinary Medicine, 102, 274- 283.

15. Gelaye, E., Belay, A., Ayelet, G., Jemberie, S., Yami, M, Loitsch, A., Tuppurainen E., Grabherr, R., Diallo, A., Euloge, C., Lamien., 2015. Capripox disease in Ethiopia: Genetic differences between field isolates and vaccine strain, and implications for vaccination failure. Antiviral Research, 119, 28-35.

16. Kitching, R.P., 2003. Vaccines for lumpy skin disease, sheep pox and goat pox. Development of Biology (Basel), 114, 161-167.

17. Lamien, C.E., Le Goff, C., Silber R., Wallace, D.B., Gulyaz, V., Tuppurainen, E., Madani, H., Caufour, P., Adam, T., El .H.M., Luckins, A.G., Albina, E., Diallo, A., 2011a. Use of the Capripoxvirus homologue of Vaccinia virus $30 \mathrm{kDa}$ RNA polymerase subunit (RPO30) gene as a novel diagnostic and genotyping target: Development of a classical PCR method to differentiate Goat poxvirus from Sheep poxvirus. Veterinary Microbiology, 149, 30-39.

18. Lamien, C.E., Lelenta, M., Goger, W., Silber, R., Tuppurainen, E., Matijevic, M., Luckins, A.G., Diallo. A., 2011 b. Real time PCR method for simultaneous detection, quantitation and differentiation of capripoxviruses. Journal of Virological Methods, 171, 134-140.

19. Lefèvre, P.C., Gourreau, J.M., 2010. Lumpy Skin disease. In: Lefèvre, P.C., Blancou, J., Chalmette, R., Uilenberg, G. (Eds.), Infectious and Parasitic diseases of Livestock. Lavoisier, Paris. Pp, 393-407.

20. Mangana-Vougiouka, P., Markoulatos, O., Koptopoulos, G., Nomikou, K., Bakandritsos, N., Papadopoulos, O., 1999. Sheep poxvirus identification by PCR in cell cultures. Journal of Virological Methods, 77, 75-79.

21. Mebratu, G., Kassa, B., Fikre, Y., Berhanu, B., 1984. Observations on the outbreak of lumpy skin disease in Ethiopia. La Revue d'Elevage et de Medicine Veterinaire des pays Tropicaux, 37, 395-399.

22. Mohamed, H.T., David, B.W., Gertruida, H.G., Roelf, R.G., Maanda, N.P., Rashied, M.A., Sultan, I.A., 2014. Lumpy skin disease of cattle: an emerging problem in the Sultanate of Oman. Tropical Animal Health and Production, 46, 241-246.

23. Moulton, L.H., Wolff, M.C., Brenneman, G., Santosham, M., 1995. Case-cohort analysis of case-coverage studies of vaccine effectiveness. American Journal of Epidemiology, 142, 1000-1006.

24. National Meteorological Service Agency (NMSA)., 2005. Seasonal Agro Meteorological Bulletin, Volume 15 No. 3, Addis Ababa, Ethiopia.

25. Office International des Epizooties (OIE)., 2010. Lumpy skin disease. In: manual of diagnostic tests and vaccines for terrestrial animals. Office International des Epizooties, World Organization for Animal Health, Paris, pp, 1-13.

26. Office International des Epizooties (OIE)., 2012. Manual of Diagnostic Tests and Vaccines for Terrestrial Animals, seventh edition. The World Organization for Animal Health (OIE), Paris.

27. Radostits, O.M., Gay, C.C., Hinchcliff, K.W., Constable, P.D., 2007. Veterinary medicine: A textbook of the diseases of cattle, horses, sheep, pigs and goats, 10th Edition, Saunders Ltd, Philadelphia, USA, 1424-1426.

28. Tamura, K., Stecher, G., Peterson, D., Filipski, A., Kumar, S., 2013. MEGA6: Molecular Evolutionary Genetics Analysis version 6.0. Molecular Biology and Evolution, 30, 2725-2729.

29. Tuppurainen, E. S., Stoltsz., W. H., Troskie, M., Wallace, D.B., Oura, C.A., Mellor, P.S., Coetzer, J.A., Venter, E.H., 2011. A potential role for ixodid (hard) tick vectors in the transmission of lumpy skin disease virus in cattle. Transboundary Emerging Disease, 58, 93-104. 
30. Tuppurainen, E.S., Pearson, C.R., Bachanek-Bankowska, K., Knowles, N.J., Amareen, S., Frost, L., Henstock, M.R., Lamien, C.E., Diallo A., Martens., P.P.C., 2014. Characterization of sheep pox virus vaccine for cattle against lumpy skin disease virus. Antiviral Research, 109, 1-6.

31. Tuppurainen, E.S.M., Oura, C.A.L., 2012. Review; Lumpy skin disease: An emerging threat to Europe, Middle East and Asia. Transboundary Emerging Disease, 59, 40-48.

32. Woods, J.A., 1988. Lumpy skin disease - A Review. Tropical Animal Health and Production, 20, 11-17. 